\title{
A driving force for change: interstitial flow as a morphoregulator
}

\author{
Joseph M. Rutkowski and Melody A. Swartz
}

Institute of Bioengineering, École Polytechnique Fédérale de Lausanne (EPFL), 1015 Lausanne, Switzerland

\begin{abstract}
Dynamic stresses that are present in all living tissues drive small fluid flows, called interstitial flows, through the extracellular matrix. Interstitial flow not only helps to transport nutrients throughout the tissue, but also has important roles in tissue maintenance and pathobiology that have been, until recently, largely overlooked. Here, we present evidence for the various effects of interstitial flow on cell biology, including its roles in embryonic development, tissue morphogenesis and remodeling, inflammation and lymphedema, tumor biology and immune cell trafficking. We also discuss possible mechanisms by which interstitial flow can induce morphoregulation, including direct shear stress, matrix-cell transduction (as has been proposed in the endothelial glycocalyx) and the newly emerging concept of autologous gradient formation.
\end{abstract}

\section{Introduction}

The importance of dynamic mechanical stress (see Glossary) in tissue development, maintenance, function and pathogenesis has been well established for several decades. The field of biomechanics originated to characterize the mechanical behavior of tissues that serve obvious mechanical functions (e.g. bone, muscle, arteries and lung tissue) and how these mechanical properties change in pathological states. This research evolved and branched into the field of mechanobiology, which focuses on understanding the cell biology that controls tissue mechanics, in other words, the response of cells to mechanical stress and the way they adapt to and control their mechanical environment [1,2]. Mechanobiology research today remains largely devoted to understanding the control of mechanically important tissues for tissue engineering applications and other areas of therapeutic design.

However, even in tissues that do not serve primarily mechanical functions or undergo obvious strains, mechanical stress is an important regulator of tissue development, health and pathology. Dynamic stresses and pressure gradients exist in all living tissues. These tissue stresses can impart forces on the cell, including fluid shear stress, pressure forces and forces on integrins, and they can also affect cell behavior by transporting solutes and shaping the extracellular distribution of key signaling proteins. For example, small fluid flows within the interstitial space are needed to drive protein transport from the blood to interstitial cells, because proteins are too large to readily

Corresponding author: Swartz, M.A. (melody.swartz@epfl.ch).

Available online 1 December 2006. diffuse the distances between blood capillaries, distances optimized for the transport of oxygen and other small molecules to cells. Dynamic stresses are therefore not only present in all living tissues, but are also required for physiological functions and tissue homeostasis. A clear example of the necessity of activity is seen in the atrophy of muscle and bone when movement is limited.

Here, we focus on the importance of a subtle but essential dynamic force: interstitial fluid flow. We argue not only that interstitial flow is an important morphoregulator in tissue development, maintenance and remodeling, but also that it is used by interstitial cells to signal the state of their surroundings, help establish extracellular microenvironments, and guide lymphocytes and tumor cells towards draining lymphatic vessels (also referred to generally herein as lymphatics).

\section{What is interstitial flow?}

Interstitial flow is fluid flow through a 3D matrix, around interstitial cells such as fibroblasts, tumor cells, tissue immune cells and adipocytes. It differs from open-channel flow, such as blood flow within vessels, in several ways (Figure 1): for example, it generally flows with a much slower velocity because of the high flow resistance of the extracellular matrix, it moves around the cell-matrix interface in all directions rather than only on the apical side, and it can have important effects on pericellular protein gradients, particularly those that are matrix binding. Interstitial flow also occurs across the blood vessel wall (called transvascular flow) (e.g. in arteries, this flow is two orders of magnitude slower than that of the blood) and the glycocalyx on the luminal surface of blood endothelium can impart some features of interstitial flow on the endothelium.

\section{Glossary}

Autologous chemotaxis: a migratory mechanism whereby a cell can respond to a chemotactic gradient while at the same time being the source of the chemokine, when interstitial flow is present, to create the transcellular gradient.

Convection: transport driven by fluid movement.

Diffusion: transport driven by random thermal molecular motion (from high to low concentration).

Fluid shear stress: the tangential stress exerted on a surface (e.g. of a cell) due to fluid viscosity and flow.

Glycocalyx: a layer of heparan sulfate proteoglycans and hyaluronan secreted by and coating endothelial cells.

Matrix binding: the ability of a morphogen to be chemically bound to the matrix, typically through interactions with sulfated proteoglycans.

Pressure gradient: the difference in pressure divided by a unit length.

Stress: force per unit area. 


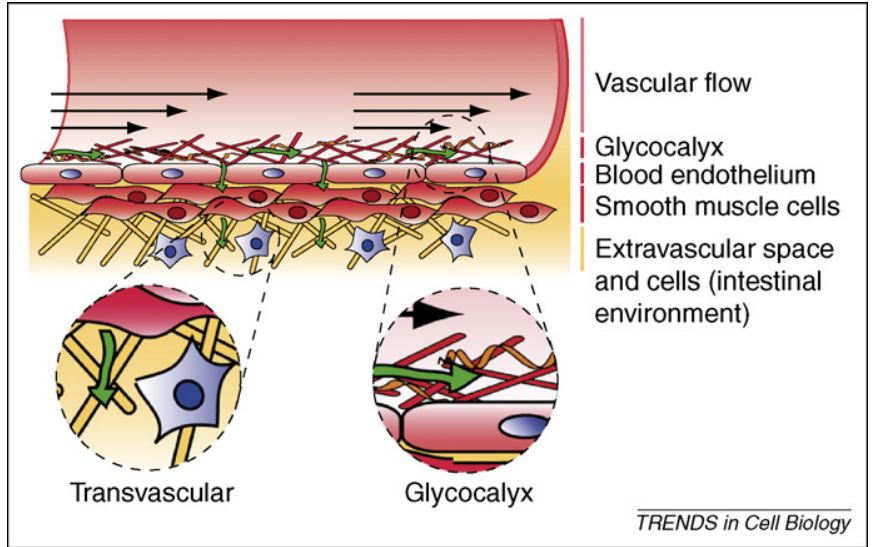

Figure 1. Interstitial and intravascular flows and their corresponding microenvironments. A blood vessel is shown, with insets showing the transvascular and glycocalyx regions. Black arrows represent luminal flow, and green arrows represent intra-glycocalyx, transvascular, and interstitial flows. By definition, interstitial flow refers to fluid flow around an interstitial cell: a cell attached to extracellular matrix in three dimensions. For endothelial cells, flow within vessels occurs only on the apical surface. Where there is a glycocalyx, flow might percolate through that network of proteoglycans and cause complex stresses on the cell surface, but fluid flow is still two-dimensional with respect to the cell surface. However, the intimate cell-matrix-flow interactions at the glycocalyx-cell interface might lead to effects similar to those in three dimensions. Intravascular pressure can drive flow through the vascular wall, but endothelial cells and smooth muscle cells will experience flow stresses through cell-cell connections and through cell-matrix connections as in true interstitial flow.

Interstitial flow is driven primarily by plasma leaving a blood capillary through its wall and draining into the initial lymphatics (Figure 2). Even when lymphatics are not functional or are blocked, some interstitial flow can occur by plasma reabsorption in post-capillary venules (although not all can be removed this way). In healthy adult tissues, the pressure difference between the two capillary networks maintains fluid pressure gradients that are altered by skeletal motion and also by subtle movements, such as those arising from arterial pulsation, respiration and organ move-

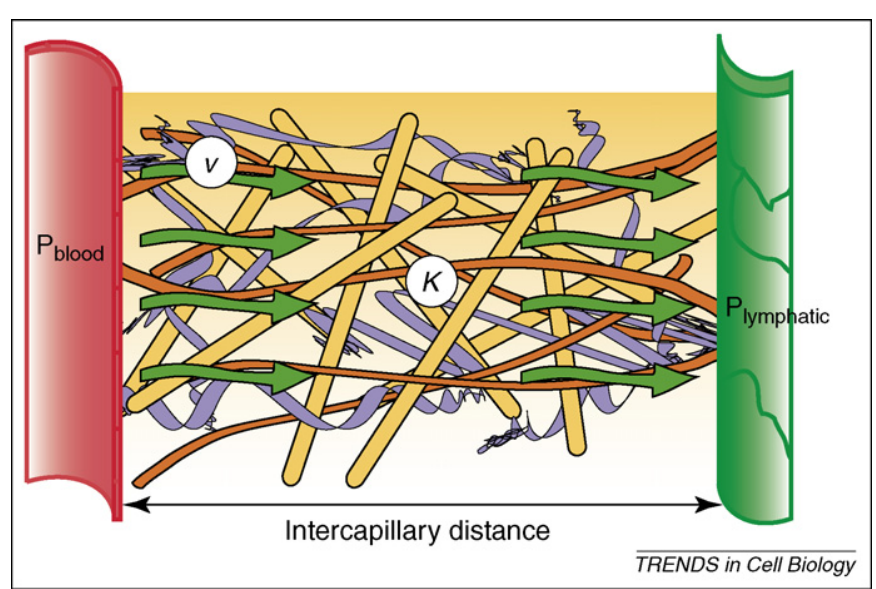

Figure 2. Determinants of interstitial flow velocity. Darcy's law describes fluid flow through a 3D matrix as being driven by a fluid pressure gradient and controlled by a flow resistance. It is analogous to water flow through a mat of hair in a bathtub drain: the more dense the hair mat, the slower the fluid drains. One important source of interstitial convection is fluid movement driven by the pressure gradient between blood and lymphatic capillaries (red and green, respectively), determined by the pressure difference $\left(P_{\text {blood }}-P_{\text {lymphatic }}\right)$ divided by the intercapillary distance. The pressure gradient yields a resultant velocity, $v$ (green arrows), controlled by the interstitial hydraulic conductivity, $K$, which varies depending on the density and composition of each tissue (Box 1, Table 1).
Box 1. Darcy's law

$$
v=-K \frac{\Delta P}{l}
$$

where $v$ is the bulk fluid velocity, $K$ is the hydraulic conductivity, and $\Delta P$ is the pressure difference over length $l$ of the tissue.

Darcy's law provides the fundamental equation for low Reynolds number flows through porous media. Originally developed in 1856 to describe flow through a gravel bed, Darcy's law (here in simplified form) can be applied to biological tissues to calculate interstitial flow velocities because the physical parameters of pressure and $K$ can be measured more readily.

ment. One noted exception to the intervascular pressure driving force is in cartilage and bone, where dynamic compression drives flow through the matrix.

There are only a few direct measurements of interstitial flow velocities in vivo in the literature; these velocities are difficult to measure and interpret because they are so slow and heterogeneous, they depend on the tracer moving with the same velocity as the fluid (which is difficult to confirm in the dense interstitial space, and the tracer can also cause artifacts when introduced into the tissue), and they have only been measured close to the surface by fluorescence recovery after photobleaching (FRAP) or nuclear magnetic resonance (NMR). Furthermore, these measurements are typically performed in anesthetized animals, in which interstitial fluid velocities are likely to be substantially different from those in awake animals because of changes in blood pressure and lymphatic pumping [3,4]. Reported measured velocities vary between 0.1 and $4.0 \mu \mathrm{m} \mathrm{s}^{-1}[5,6]$. Interstitial flow velocity can also be estimated using Darcy's law, which relates velocity to the pressure gradient and the hydraulic conductivity, $K$ (Box 1 ). This is sometimes more convenient, because interstitial pressures can be measured more reliably using micropipettes or wick-in-needle techniques [7] and $K$ can be measured in tissues ex vivo using confined compression tests $[8,9]$.

Even though it can be extremely slow, interstitial flow can have important effects on tissue morphogenesis and function, cell migration and differentiation and matrix remodeling, among other processes. The mechanisms by which such flows can drive cell response might be purely mechanical, such as shear stress on the cell surface, pressure force 'pushing' on the cell or tethering forces on cellmatrix connections (Figure 3). Importantly, it can also have non-mechanical affects on the cells, such as shifting the pericellular distribution of secreted proteins (e.g. morphogens, proteases and chemokines). These transport effects are likely to be more important than mechanical stress in tissues that do not serve primarily mechanical functions, because of the small magnitudes of stresses and flows found there. In these tissues, interstitial flow-induced protein redistribution might help to direct cell migration and guide the cell-cell interactions that lead to pattern formation during morphogenesis. Thus, interstitial flow affects both the mechanical microenvironment of the cell and the biochemical environment to which it is so acutely tuned.

\section{Biological flows in development}

In embryos that have not yet developed a vascular system or heart, flow that is driven through the differentiating cell 


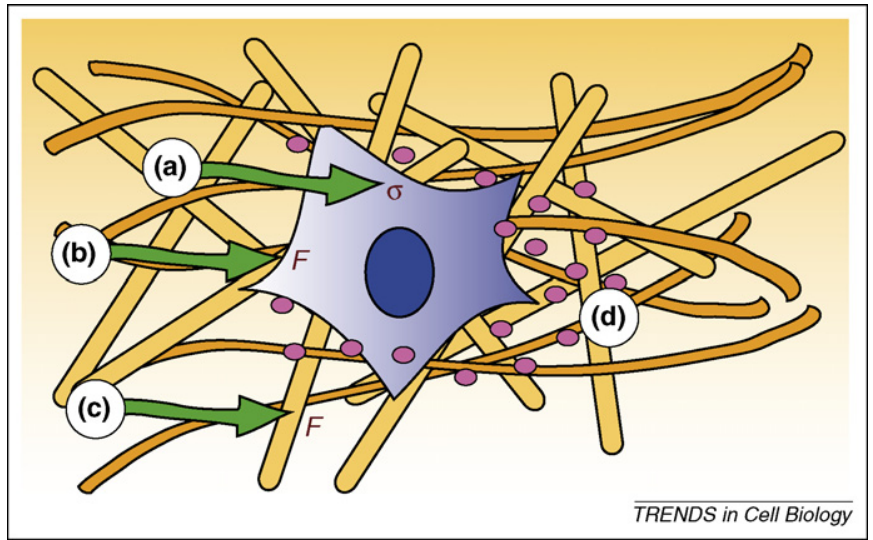

Figure 3. The direct effects that interstitial flow has on cells. Interstitial flow can induce (a) fluid shear stress, $\sigma$, on the cell surface; (b) forces normal to the cell surface $(F)$; (c) shear and normal forces to the pericellular matrix that is mechanically coupled to the cytoskeleton; and (d) redistribution of pericellular proteins (autocrine and paracrine signals) that bind cell receptors.

mass is necessary for proper development. In the embryonic node (an embryonic structure located at the anterior tip of the primitive centerline) ciliary movement generates the leftward movement of fluid that leads to the left-right asymmetry of the organs (in which the heart is on the left, the liver on the right, etc.) [10], and when this cilia-driven flow within the node is reversed experimentally, the left-right asymmetry becomes reversed [11]. It has been suggested through mathematical modeling that nodal flow directs morphogen transport and mixing, thus driving asymmetric development [12]. This is indeed likely, because the actions of morphogens (to give cells directional and positional information) are achieved through their transcellular concentration gradients rather than their absolute amounts. In developing embryos, such spatial information guides cell differentiation [13]. Morphogen transport and gradient patterning are also believed to regulate the branching of developing lungs in the embryo [14], and it is expected that interstitial flows, caused by embryonic lung movements that simulate breathing, would influence morphogen distributions. Interstitial flow can also impart shear stress on the cell surface, which, as a mechanical stimulus, can itself drive embryonic cell differentiation and determine lineage fate $[15,16]$ and could be responsible for shaping organs $[17,18]$.

Interstitial flow has also been implicated in lymphatic development. Using a skin regeneration model in which interstitial fluid flow could be traced and correlated with lymphatic proliferation, migration and reorganization over time, it has been shown that lymphatic cells migrate in the direction of interstitial flow and organize around fluid channels, and that they cannot organize into functional capillaries when interstitial flow is severely reduced $[19,20]$. Thus, interstitial flow can act as an important morphogenic cue, by mechanisms we discuss later.

\section{Interstitial flow in tissue function and pathology}

Much evidence has emerged to indicate that interstitial flow has an important regulatory role in tissue function. Transvascular flow across the arterial wall provides nutrient transport to the metabolically active cells there, and seemingly has a crucial role in maintaining arterial smooth muscle tone [21-23]. In cartilage, where lymphatics are absent and intercellular distances are large, interstitial fluid flow is driven by mechanical loading and is necessary for nutrient transport and cell-cell communication when diffusion is inadequate [24-26]. Also, interstitial flow rather than solid stress is responsible for at least some of the mechanical stress-induced matrix production in cartilage [27], because dynamic rather than static compression was found to promote proteoglycan and collagen synthesis [28] and increase chondrocyte metabolism [29] (interstitial flow is always present in tissues undergoing dynamic compression). Dynamic compression stimulates directional deposition of proteoglycans and matrix fiber compaction in the direction of flow [26] and directs remodeling by enhancing the transport of tissue inhibitor of metalloproteinase-1 (TIMP-1) [30]. In bone, physical activity causes oscillatory compression that has been estimated to increase the convective transport of macromolecules up to 100 times more than what is possible with diffusion [31]. Finally, in tissue repair, the migration of endothelial and epithelial cells is crucial for wound healing, which (at least on $2 \mathrm{D}$ surfaces) can be activated by shear stress [32,33], suggesting that interstitial flow also promotes wound healing in 3D tissues by shear stress. In vitro, interstitial flow enhances blood and lymphatic capillary formation [34] and acts synergistically with matrix-bound VEGF to induce capillarogenesis, probably by enhancing and directing the liberation of VEGF from the matrix to guide organization in the direction of flow [35-37].

Physiological evidence that interstitial flow is crucial in normal tissue function can be seen in pathologies in which interstitial flow is reduced or enhanced. Lymphedema is a condition in which interstitial fluid flow is severely reduced due to either malformations in the lymphatic system (primary lymphedema) or blockage downstream, such as that which occurs after lymph node resection (secondary lymphedema). The accumulation, rather than clearance, of fluid from the interstitial space results in inflammation and extensive tissue remodeling, lymphatic hyperplasia, and adipocyte growth and lipid accumulation [38,39]. Fluid stagnation in lymphedema also prevents normal immune cell trafficking in the affected tissue, which can exacerbate the pathology. These resultant chronic pathological conditions in lymphedema highlight the importance of interstitial fluid convection in maintaining healthy tissue.

Abnormally increased interstitial flow rates can occur during inflammation (when blood capillaries become leaky) and can also trigger fibroblasts to differentiate or remodel the extracellular matrix. Although this can be due to increased transport of differentiation factors from inflammatory cells to fibroblasts, increased interstitial flow itself could be an important contributing factor to the development of tissue fibrosis: in vitro studies have shown that 4$10 \mu \mathrm{m} \mathrm{s}^{-1}$ interstitial flow through a 3D collagen matrix seeded with human lung or dermal fibroblasts causes autocrine upregulation of transforming growth factor (TGF)- $\beta 1$, differentiation into myofibroblasts (Figure 4a), increased collagen production and collagen alignment [4042]. In this way, high interstitial flow could be an early signaling cue of inflammation that triggers fibroblasts to begin rapid matrix repair. It could also help explain why 


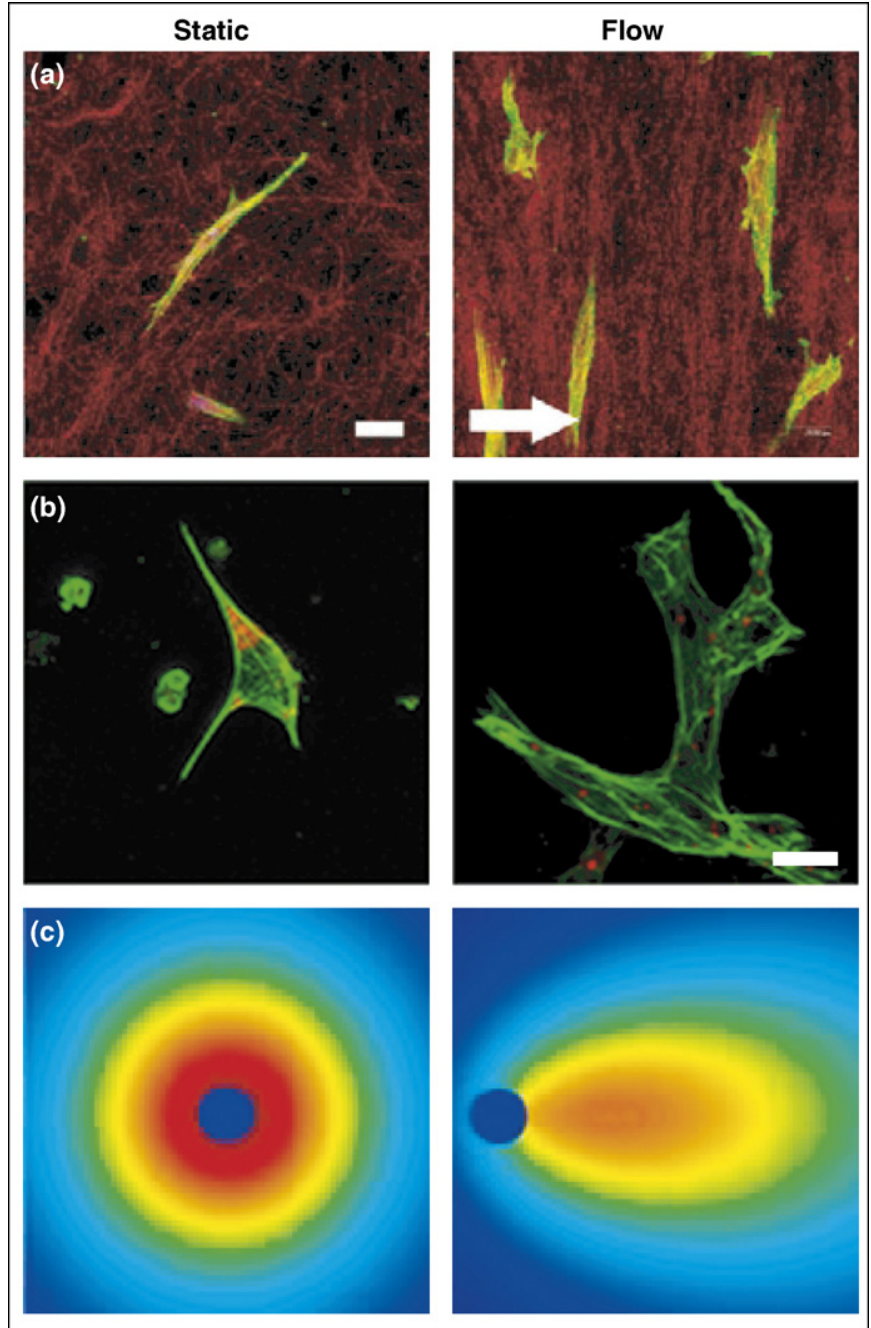

Figure 4. Examples of cellular responses to interstitial flow. (a) Interstitial flow a $4 \mu \mathrm{m} / \mathrm{s}$ levels (right) cause human dermal fibroblasts seeded in type I collagen to differentiate into myofibroblasts by upregulation of TGF- $\beta$ and align themselves and the matrix fibers perpendicular to the flow. Reproduced with permission from Ref. [41]; the scale bar represents $20 \mu \mathrm{m}$. (b) Human blood endothelial cells form branched structures with lumens when cultured under interstitial flow (right). Here, low levels $(4 \mu \mathrm{m} / \mathrm{s})$ of interstitial flow greatly enhanced the effects of matrix-bound VEGF on capillary morphogenesis. Reproduced with permission from Ref. [35]; the scale bar represents $40 \mu \mathrm{m}$. (c) Computational modeling demonstrates that when morphogens or chemokines are autologously secreted by a cell in matrix-binding form and under low levels of interstitial flow, autologous morphogen gradients develop to guide cell processes in the direction of flow. The concentration profiles of liberated VEGF released by cell-secreted proteases, under the conditions in (b) are shown. Reproduced with permission from Ref. [35].

tissue fibrosis often follows inflammation in many tissues, including the lung, skin and surrounding tumors, and why fibrosis can occur in the apparent absence of inflammatory cells, such as in idiopathic pulmonary fibrosis [43,44].

\section{Interstitial flow in cancer}

Interstitial flow in and around tumor tissue has particular importance in delivering anticancer agents to tumor tissue. Given that growing tumors induce angiogenesis and angiogenic tumor vessels are more permeable to proteins and large molecules than mature vessels [45], there has been substantial interest in exploiting tumor vessel permeability to selectively accumulate drug carriers by size in tumor tissue. However, interstitial transport is driven by pressure differences between the blood and interstitium, and in tumors interstitial fluid pressure is higher, ranging from $10 \mathrm{mmHg}$ to $20 \mathrm{mmHg}$ (with measurements as high as $90 \mathrm{mmHg}$ ), than in normal tissues, which have pressures that are typically $<10 \mathrm{mmHg}$ (Table 1) [46]. Thus, the driving force for fluid movement from the blood into the tumor stroma is lower than that in normal tissues. These high interstitial pressures are due in part to the lack of functional lymphatics within the tumor [47] and result in a net convective flow out from the tumor mass into the surrounding tissue as a result of the lower interstitial pressure found there. (The conclusion that tumors lack functional lymphatics is controversial, however; several reports have suggested that metastatic tumors can induce lymphatic growth into the tumor mass [48].)

Although the challenges to therapeutic delivery to solid tumors have been researched extensively, the impact of the extratumoral interstitial flow environment on tumor biology has not been much explored. Slow interstitial flow from the tumor mass into the surrounding tissue and draining lymphatics might, for example, help the tumor invade tissue and lymphatics by directing proteolytic enzymes and autocrine chemokines away from the tumor and towards the draining lymphatics. Alternatively, it might promote the formation of a fibrotic capsule around the tumor (as flow itself can drive fibroblast differentiation, as mentioned earlier [41]), which might inhibit tumor spread. Also, it is likely to affect the recruitment and function of tumorassociated macrophages that are found around highly invasive tumors [49] by further distributing chemotactic factors.

New insights into how interstitial flow can affect tumor biology and invasion are just beginning to emerge. For example, it was recently shown that tumor cell proliferation can be influenced by intratumoral pressure [50]. In tumors overexpressing lymphangiogenic growth factors, peritumoral lymphatics (those surrounding and draining the tumor) were found to drain fluid at an increased rate, and tumor cells were directly observed homing to those lymphatics [51]. Finally, recent in vitro studies suggest that interstitial flow from tumor cells directed towards lymphatic endothelial cells greatly enhances the migration of tumor cells towards the lymphatics, through a combination of autocrine and paracrine signaling mechanisms [Shields, J.D, unpublished data]. We explore this possibility further when discussing autologous chemotaxis.

\section{Mechanisms of flow-induced cell response}

Evidence that interstitial flow can direct mechanotransduction events on the cell surface comes from recent studies on the endothelial glycocalyx. Originally, the shear stress effects seen on endothelial cells were presumed to be due directly to fluid shear stress acting on the cell surface, but there is increasing appreciation for the role of the glycocalyx in moderating or amplifying fluid stresses to the cell surface. The primary constituents of the glycocalyx are heparan sulfate, chondroitin sulfate and hyaluronan [52], anchored to the cell by proteoglycan core proteins. The role of the glycocalyx in transducing shear stress to the cell has been demonstrated by selectively degrading these components and exposing the cells to shear: for example, when either heparinase or hyaluronidase were applied to an endothelial cell monolayer, well established responses to shear stress, such as the release of nitric oxide, were 
Table 1. Physical characteristics of interstitial flows in various tissues ${ }^{a}$

\begin{tabular}{|c|c|c|c|c|c|}
\hline \multirow[t]{2}{*}{ Tissue } & (a) & (b) & (c) & (d) & \multirow[t]{2}{*}{ Refs } \\
\hline & $v_{\text {measured }}\left(\mu \mathrm{m} \mathrm{s}^{-1}\right)$ & $\begin{array}{l}\text { Hydraulic conductivity, } \\
K\left(\times 10^{-9} \mathrm{~cm}^{2} \mathrm{mmHg} \text { s }\right)\end{array}$ & $\begin{array}{l}\text { Estimated pressure gradient } \\
\left(\mathrm{mmHg} 100 \mu \mathrm{m}^{-1}\right)\end{array}$ & $v_{\text {calculated }}{ }^{b}\left(\mu \mathrm{m} \mathrm{s}^{-1}\right)$ & \\
\hline Tumor capsule & $0.1-0.5$ & & & & (a) $[6,46]$ \\
\hline Granulation tissue (rabbit) & $0.59 \pm 0.16$ & & & & (a) [5] \\
\hline \multirow[t]{2}{*}{$\begin{array}{l}\text { Neoplastic tissue (tumor } \\
\text { xenograft) }\end{array}$} & $0.55 \pm 0.16$ & $92-2480$ & & & (a) [5] \\
\hline & & & & & (b) [59] \\
\hline \multirow[t]{2}{*}{ Skin } & & $1500-1680$ & $0.5-6.0$ & $0.7-10.1$ & (b) $[9,60]$ \\
\hline & & & & & (c) $[46,61]$ \\
\hline \multirow[t]{2}{*}{ Mesentery } & & $41-253$ & 2.4 & $0.1-0.6$ & (b) $[9]$ \\
\hline & & & & & (c) $[62]$ \\
\hline \multirow[t]{3}{*}{ Aorta, media and intima } & & $0.3-0.7$ & $42-152$ & $0.013-0.106$ & (b) $[9,63]$ \\
\hline & & & & & (c) $[63]$ \\
\hline & & & & & (d) $[63]$ \\
\hline Femoral head cartilage & & $0.1-0.2$ & & & (b) [9] \\
\hline Corneal stroma & & $0.71-1.60$ & & & (b) [9] \\
\hline
\end{tabular}

There have been few measurements of interstitial flow velocities in vivo, so the more accessible parameters of tissue hydraulic conductivity and interstitial pressure can be used to estimate velocities using Darcy's Law (Box 1). Tissues that experience high pressures and for which homeostasis demands low flow across them, such as the aorta, have the lowest conductivities to prevent flow.

${ }^{\text {b } C a l c u l a t e d ~ u s i n g ~ r e p o r t e d ~} K$ and estimated pressure gradient.

eliminated [53,54]. Although such stresses are not identical to those in true interstitial flow, as mentioned earlier, the fact that cells might sense flow only when the glycocalyx is intact gives strong support to the importance of cell-ECM connections in transducing mechanical stress by slow interstitial flow. In 3D in vitro matrices, cell surface shear stresses for flow rates of $1 \mu \mathrm{m} \mathrm{s}^{-1}$ were estimated to average $5 \times 10^{-3}$ to $7 \times 10^{-3}$ dyne $\mathrm{cm}^{-2}$ and peak at $1.5 \times 10^{-2}$ dyne $\mathrm{cm}^{-2}$, although the precise architecture of matrix fibers strongly affected these stresses [55]. It is unknown to what limit cells might sense shear stress, but with such low levels, it is likely that the glycocalyx and/or surrounding ECM helps amplify the signals to transduce mechanical stress to the cell.

Another mechanism by which interstitial flow can affect cellular responses is by changing pericellular diffusion gradients of morphogens that are redistributed by the flow according to how they are transported: by diffusive and/or convective transport (see Glossary). Given that morphogens act by directing cellular responses spatially according to transcellular gradients, subtle changes in their pericellular distributions (e.g. the introduction of asymmetry) can have important effects on directed cell processes. In addition, many chemokines and growth factors that are both secreted by and act upon the same cell bind strongly to the matrix (usually to sulfated proteoglycan components of the matrix), including members of the vascular endothelial growth factor (VEGF), fibroblast growth factor (FGF), Wnt and TGF families and many immunoresponsive chemokines, such as CCL21, that direct leukocyte migration [56]. The binding of these factors to the matrix gives the cell more control of its microenvironment and enables solid-phase gradients to form. In addition, some of these proteins can signal to cells in both their liberated and their matrix-bound forms.

\section{Interstitial flow in cell homing: the concept of autologous chemotaxis}

Chemokine gradients act as directional signals for cell chemotaxis or morphogenesis. Because migrating cells move up a chemokine concentration gradient, it is generally assumed that the chemokine is secreted by an upstream cell or tissue. An alternative mechanism, however, has recently been described whereby a cell can receive directional cues while simultaneously being the source of such cues, using interstitial flow. In this mechanism, even extremely subtle flows can affect the pericellular distribution of self-secreted proteins that interact with the matrix and thus cause autologous transcellular gradients, increasing in the direction of flow, to form (Figure 4c) [57]. Flow only slightly biases the distributions of a secreted matrix-binding morphogen and proteases that can liberate the morphogen, but those effects multiply and combine with the fact that, once the morphogen is liberated, it is further biased by flow. In this way, the ability of the secreted morphogen to bind the matrix serves as an amplification mechanism for autocrine gradient formation only in the presence of subtle interstitial flow. This has been demonstrated experimentally using a $3 \mathrm{D}$ culture of endothelial cells suspended in a VEGF-containing matrix, in which VEGF was covalently bound and liberated only upon proteolytic release by the cells [35]; capillary organization occurred only in the presence of both VEGF and interstitial flow (Figure 4b). This was presumably due to directed liberation of VEGF.

This putative phenomenon of autocrine morphogen gradient formation by interstitial flow and matrix binding of morphogens suggests that leukocytes and tumor cells might use interstitial flow to home to draining lymphatics. This is possible when the cell expresses the homing chemokine receptor and also secretes the chemokine ligand. These cells include tumor cells [expressing the chemokine receptor CCR7 and the ligand CCL21 (Shields, J.D., unpublished data)], dendritic cells (expressing the chemokine receptor CCR7 and the ligand CCL19 [58]) and macrophages (expressing VEGF receptor-3 and its ligand VEGF-C [49]). As all of these ligands can bind to the matrix, and as all are important cues for migration, there is potential for each to direct migration by this mechanism. Mathematical modeling shows that even the smallest flows can create autologous gradients in such systems [57]. Not only might this help to explain why certain cell types have receptors for ligands that they 
themselves secrete, but this mechanism of self-directed cell migration might also be fundamental to the movement of tumor and immune cells in the direction of interstitial transport, that is, towards functional lymphatic vessels and on to the nearest lymph node. Flow-induced autologous chemotaxis has recently been demonstrated in vitro, using human invasive and noninvasive breast cancer cells, to take place through the chemokine CCR7 and its receptor CCL21: whereas flow strongly enhanced the migration of tumor cells through 3D matrices in the direction of flow, blocking CCR7 signaling eliminated this effect (Shields, J.D., unpublished data). Thus, it is possible that autologous chemotaxis is a powerful mechanism that cells use to find and home to functional draining lymphatics.

\section{Concluding remarks}

In summary, interstitial flow is an important component of normal tissue function and homeostasis and of many pathologies, from development through to adulthood. It might also be a key morphoregulator, acting by giving directional cues to cells. As prime examples, dendritic cell trafficking to lymph nodes and tumor invasion and dissemination through lymphatics can use interstitial flow to home towards lymphatic capillaries. Future research that incorporates and examines interstitial flow as a key microenvironmental component will be necessary to elucidate such mechanisms fully and exploit its potential in therapy and tissue engineering.

\section{References}

1 Pedersen, J.A. and Swartz, M.A. (2005) Mechanobiology in the third dimension. Ann. Biomed. Eng. 33, 1469-1490

2 Wang, J.H. and Thampatty, B.P. (2006) An introductory review of cell mechanobiology. Biomech. Model. Mechanobiol. 5, 1-16

3 Bruegger, D. et al. (2002) Microvascular changes during anesthesia: sevoflurane compared with propofol. Acta Anaesthesiol. Scand. 46, 481-487

4 McHale, N.G. and Thornbury, K. (1986) A method for studying lymphatic pumping activity in conscious and anaesthetized sheep. J. Physiol. 378, 109-118

5 Chary, S.R. and Jain, R.K. (1989) Direct measurement of interstitial convection and diffusion of albumin in normal and neoplastic tissues by fluorescence photobleaching. Proc. Natl. Acad. Sci. U. S. A. 86, 53855389

6 Dafni, H. et al. (2002) Overexpression of vascular endothelial growth factor 165 drives peritumor interstitial convection and induces lymphatic drain: magnetic resonance imaging, confocal microscopy, and histological tracking of triple-labeled albumin. Cancer Res. 62, 6731-6739

7 Wiig, H. (1990) Evaluation of methodologies for measurement of interstitial fluid pressure $(\mathrm{Pi})$ : physiological implications of recent $\mathrm{Pi}$ data. Crit. Rev. Biomed. Eng. 18, 27-54

8 Anand, S. et al. (1995) Enzyme-mediated proteolysis of fibrous biopolymers - dissolution front movement in fibrin or collagen under conditions of diffusive or convective-transport. Biotechnol. Bioeng. 48, 89-107

9 Levick, J.R. (1987) Flow through interstitium and other fibrous matrices. Q. J. Exp. Physiol. 72, 409-437

10 Mercola, M. (2003) Left-right asymmetry: nodal points. J. Cell Sci. 116, $3251-3257$

11 Nonaka, S. et al. (2002) Determination of left-right patterning of the mouse embryo by artificial nodal flow. Nature 418, 96-99

12 Cartwright, J.H. et al. (2004) Fluid-dynamical basis of the embryonic development of left-right asymmetry in vertebrates. Proc. Natl. Acad. Sci. U. S. A. 101, 7234-7239

13 Gurdon, J.B. and Bourillot, P.Y. (2001) Morphogen gradient interpretation. Nature 413, 797-803
14 Warburton, D. et al. (2005) Molecular mechanisms of early lung specification and branching morphogenesis. Pediatr. Res. 57, 26R$37 \mathrm{R}$

15 Estes, B.T. et al. (2004) Mechanical signals as regulators of stem cell fate. Curr. Top. Dev. Biol. 60, 91-126

16 Hosseinkhani, H. et al. (2005) Perfusion culture enhances osteogenic differentiation of rat mesenchymal stem cells in collagen sponge reinforced with poly(glycolic acid) fiber. Tissue Eng. 11, 1476-1488

17 Blatnik, J.S. et al. (2005) The influence of fluid shear stress on the remodeling of the embryonic primary capillary plexus. Biomech. Model. Mechanobiol. 4, 211-220

18 DeGroff, C.G. et al. (2003) Flow in the early embryonic human heart: a numerical study. Pediatr. Cardiol. 24, 375-380

19 Boardman, K.C. and Swartz, M.A. (2003) Interstitial flow as a guide for lymphangiogenesis. Circ. Res. 92, 801-808

20 Rutkowski, J.M. et al. (2006) Characterization of lymphangiogenesis in a model of adult skin regeneration. Am. J. Physiol. Heart Circ. Physiol. 291, H1402-H1410

21 Wang, S. and Tarbell, J.M. (2000) Effect of fluid flow on smooth muscle cells in a 3-dimensional collagen gel model. Arterioscler. Thromb. Vasc. Biol. 20, 2220-2225

22 Tada, S. and Tarbell, J.M. (2000) Interstitial flow through the internal elastic lamina affects shear stress on arterial smooth muscle cells. Am. J. Physiol. Heart Circ. Physiol. 278, H1589-H1597

$23 \mathrm{Kim}, \mathrm{M} . \mathrm{H}$. et al. (2004) Control of the arteriolar myogenic response by transvascular fluid filtration. Microvasc. Res. 68, 30-37

24 Grodzinsky, A.J. et al. (2000) Cartilage tissue remodeling in response to mechanical forces. Annu. Rev. Biomed. Eng. 2, 691-713

25 Chandran, P.L. and Barocas, V.H. (2004) Microstructural mechanics of collagen gels in confined compression: poroelasticity, viscoelasticity, and collapse. J. Biomech. Eng. 126, 152-166

26 Quinn, T.M. et al. (1998) Mechanical compression alters proteoglycan deposition and matrix deformation around individual cells in cartilage explants. J. Cell Sci. 111, 573-583

27 Fitzgerald, J.B. et al. (2006) Shear and compression differentially regulate clusters of functionally-related temporal transcription patterns in cartilage tissue. J. Biol. Chem. 281, 2409-24103

28 Kerin, A. et al. (2002) Molecular basis of osteoarthritis: biomechanical aspects. Cell. Mol. Life Sci. 59, 27-35

29 Evans, R.C. and Quinn, T.M. (2006) Dynamic compression augments interstitial transport of a glucose-like solute in articular cartilage. Biophys. J. 91, 1541-1547

30 Garcia, A.M. et al. (1998) Transport of tissue inhibitor of metalloproteinases-1 through cartilage: contributions of fluid flow and electrical migration. J. Orthop. Res. 16, 734-742

31 Knothe Tate, M.L. (2003) Whither flows the fluid in bone? An osteocyte's perspective. J. Biomech. 36, 1409-1424

32 Albuquerque, M.L. et al. (2000) Shear stress enhances human endothelial cell wound closure in vitro. Am. J. Physiol. Heart Circ. Physiol. 279, H293-H302

$33 \mathrm{Li}$, S. et al. (2005) Mechanotransduction in endothelial cell migration. J. Cell. Biochem. 96, 1110-1126

$34 \mathrm{Ng}$, C.P. et al. (2004) Interstitial flow differentially stimulates blood and lymphatic endothelial cell morphogenesis in vitro. Microvasc. Res. $68,258-264$

35 Helm, C.L. et al. (2005) Synergy between interstitial flow and VEGF directs capillary morphogenesis in vitro through a gradient amplification mechanism. Proc. Natl. Acad. Sci. U. S. A. 102, 15779-15784

36 Helm, C.L. et al. (2007) Engineered blood and lymphatic capillaries in 3D VEGF-fibrin-collagen matrices with interstitial flow. Biotechnol Bioeng., DOI: 10.1002/bit.21185 (http://www.interscience.wiley.com)

37 Semino, C.E. et al. (2006) Autocrine EGF receptor activation mediates endothelial cell migration and vascular morphogenesis induced by VEGF under interstitial flow. Exp. Cell Res. 312, 289-298

38 Olszewski, W.L. (2003) The lymphatic system in body homeostasis: physiological conditions. Lymphat. Res. Biol. 1, 11-21

39 Rutkowski, J.M. et al. (2006) Secondary lymphedema in the mouse tail: Lymphatic hyperplasia, VEGF-C upregulation, and the protective role of MMP-9. Microvasc Res

$40 \mathrm{Ng}$, C.P. and Swartz, M.A. (2003) Fibroblast alignment under interstitial fluid flow using a novel 3-D tissue culture model. Am. J. Physiol. Heart Circ. Physiol. 284, H1771-H1777 
$41 \mathrm{Ng}$, C.P. et al. (2005) Interstitial fluid flow induces myofibroblast differentiation and collagen alignment in vitro. J. Cell Sci. 118, $4731-4739$

$42 \mathrm{Ng}$, C.P. and Swartz, M.A. (2006) Mechanisms of interstitial flowinduced remodeling of fibroblast-collagen cultures. Ann. Biomed. Eng. 34, 446-454

43 Pardo, A. and Selman, M. (2002) Idiopathic pulmonary fibrosis: new insights in its pathogenesis. Int. J. Biochem. Cell Biol. 34, 1534-1538

44 Thannickal, V.J. et al. (2004) Mechanisms of pulmonary fibrosis. Annu. Rev. Med. 55, 395-417

45 Jain, R.K. (2005) Antiangiogenic therapy for cancer: current and emerging concepts. Oncology 19, 7-16

46 Jain, R.K. (1999) Transport of molecules, particles, and cells in solid tumors. Annu. Rev. Biomed. Eng. 1, 241-263

47 Padera, T.P. et al. (2002) Lymphatic metastasis in the absence of functional intratumor lymphatics. Science 296, 1883-1886

48 Achen, M.G. et al. (2006) Targeting lymphangiogenesis to prevent tumour metastasis. Br. J. Cancer 94, 1355-1360

49 Skobe, M. et al. (2001) Concurrent induction of lymphangiogenesis, angiogenesis, and macrophage recruitment by vascular endothelial growth factor-C in melanoma. Am. J. Pathol. 159, 893-903

50 Hofmann, M. et al. (2006) Lowering of tumor interstitial fluid pressure reduces tumor cell proliferation in a xenograft tumor model. Neoplasia 8, 89-95

51 Hoshida, T. et al. (2006) Imaging steps of lymphatic metastasis reveals that vascular endothelial growth factor-C increases metastasis by increasing delivery of cancer cells to lymph nodes: therapeutic implications. Cancer Res. 66, 8065-8075

52 Tarbell, J.M. and Pahakis, M.Y. (2006) Mechanotransduction and the glycocalyx. J. Intern. Med. 259, 339-350
53 Florian, J.A. et al. (2003) Heparan sulfate proteoglycan is a mechanosensor on endothelial cells. Circ. Res. 93, e136-e142

54 Moon, J.J. et al. (2005) Role of cell surface heparan sulfate proteoglycans in endothelial cell migration and mechanotransduction. J. Cell. Physiol. 203, 166-176

55 Pedersen, J.A. et al. (2006) Effects of extracellular fiber architecture on cell membrane shear stress in a 3D fibrous matrix. J. Biomech., DOI: 10.1016/j.jbiomech.2006.06.023

56 Patel, D.D. et al. (2001) Chemokines have diverse abilities to form solid phase gradients. Clin. Immunol. 99, 43-52

57 Fleury, M.E. et al. (2006) Autologous morphogen gradients by subtle interstitial flow and matrix interactions. Biophys. J. 91, 113121

58 Randolph, G.J. et al. (2005) Dendritic-cell trafficking to lymph nodes through lymphatic vessels. Nat. Rev. Immunol. 5, 617-628

59 Netti, P.A. et al. (2000) Role of extracellular matrix assembly in interstitial transport in solid tumors. Cancer Res. 60, 2497-2503

60 Swartz, M.A. et al. (1999) Mechanics of interstitial-lymphatic fluid transport: theoretical foundation and experimental validation. J. Biomech. 32, 1297-1307

61 Wiig, H. et al. (2003) New and active role of the interstitium in control of interstitial fluid pressure: potential therapeutic consequences. Acta Anaesthesiol. Scand. 47, 111-121

62 Kajimura, M. et al. (2001) Interstitial fluid pressure surrounding rat mesenteric venules during changes in fluid filtration. Exp. Physiol. 86, 33-38

63 Wang, D.M. and Tarbell, J.M. (1995) Modeling interstitial flow in an artery wall allows estimation of wall shear stress on smooth muscle cells. J. Biomech. Eng. 117, 358-363

\section{Have you contributed to an Elsevier publication? Did you know that you are entitled to a $30 \%$ discount on books?}

A 30\% discount is available to all Elsevier book and journal contributors when ordering books or standalone CD-ROMs directly from us.

To take advantage of your discount:

1. Choose your book(s) from www.elsevier.com or www.books.elsevier.com

\section{Place your order}

Americas:

Phone: +18007824927 for US customers

Phone: +18004603110 for Canada, South and Central America customers

Fax: +13144534898

author.contributor@elsevier.com

All other countries:

Phone: +44(0)1865 474010

Fax: $+44(0) 1865474011$

directorders@elsevier.com

You'll need to provide the name of the Elsevier book or journal to which you have contributed.

Shipping is free on prepaid orders within the US.

If you are faxing your order, please enclose a copy of this page.

\section{Make your payment}

This discount is only available on prepaid orders. Please note that this offer does not apply to multi-volume reference works or Elsevier Health Sciences products.

For more information, visit www.books.elsevier.com 